\title{
Fatores predisponentes para hipoglicemia: aumentando a segurança do paciente crítico que utiliza insulina intravenosa
}

\author{
Predisposing factors for hypoglycemia: security measures for critical patients on \\ intravenous insulin
}
Factores predisponentes para hipoglucemia: aumentando la seguridad del paciente crítico que utiliza la insulina intravenosa

\author{
Carina Teixeira Paixão ${ }^{I}$; Raquel de Mendonça Nepomuceno ${ }^{I I} ;$ Manassés Moura dos Santos ${ }^{I I I}$; Lolita Dopico da Silva ${ }^{I V}$
}

\begin{abstract}
RESUMO: Pesquisa com o objetivo de identificar os fatores predisponentes para hipoglicemia nos pacientes críticos que receberam infusão contínua de insulina. Estudo documental, retrospectivo, se rastrearam 550 prontuários dos anos de 2012 e 2013 de pacientes do pós-operatório de cirurgia cardíaca, dos quais 168 receberam insulina intravenosa e 74 apresentaram hipoglicemia grave. Os resultados principais apontam que entre os pacientes que apresentaram hipoglicemia grave, a maioria era do sexo masculino $(60,11 \%)$, idosos ( $\mathrm{md}=61,78)$, com sobrepeso $(26,75 \mathrm{Kg} / \mathrm{m} 2$,$) , hipertensos (78,57 \%)$ e isquêmicos $(52,38 \%)$. Os fatores predisponentes encontrados para hipoglicemia,com significado estatístico foram ser diabético(OR=3,6), ter insuficiência renal $(O R=5,4)$, em uso de aminas vasoativas $(O R=3,1)$. Conclui-se que uma medida de segurança, para pacientes com estes fatores, é a coleta de amostras de sangue venoso e o uso de hemogasometros para a aferição da medida glicêmica. Palavras-Chave: Insulina; glicemia; hipoglicemia; enfermagem.
\end{abstract}

\begin{abstract}
Documentary, retrospective cross research aiming at describing the predisposing factors studied in critically ill patients receiving continuous insulin infusion. Tracking was made of five hundred and fifty (550) medical records for the years 2012 and 2013 of post-operative cardiac surgery patients who received intravenous insulin. Main results show that nearly half of the patients had at least one record of hypoglycemia, the majority were male $(60.11 \%)$, elderly $(\mathrm{md}=61.78)$, overweight $(26,75 \mathrm{Kg} / \mathrm{m} 2)$, hypertense $(78.57 \%)$, and ischemic $(52.38 \%)$. Predisposing factors for hypoglycemia, with statistical significance identified among patients were as follows: diabetes $(\mathrm{OR}=3.6)$; renal failure $(\mathrm{OR}=5.4)$; and use of vasoactive amines $(\mathrm{OR}=3.1)$. Conclusions identified security actions for patients in those conditions such as venous blood collection as well as the use of blood meters to assess glycemic levels. Keywords: Insulin; glucose; hypoglycemia; nursing.
\end{abstract}

RESUMEN: Investigación con el objetivo de identificar los factores predisponentes para hipoglucemia en los pacientes críticos que recibieron infusión continua de insulina. Estudio documental, retrospectivo, se rastrearon 550 historiales de los años 2012 a 2013 de pacientes del posoperatorio de cirugía cardíaca, de los cuales 168 recibieron insulina intravenosa y 74 presentaron hipoglucemia grave. Los resultados principales apuntan que entre los pacientes que presentaron hipoglucemia grave, la mayoría era del sexo masculino $(60,11 \%)$, ancianos $(M D=61,78)$, con sobrepeso $(26,75 \mathrm{Kg} / \mathrm{m} 2)$, hipertensos $(78,57 \%)$ e isquémicos $(52,38 \%)$. Los factores predisponentes encontrados para hipoglucemia, con significado estadístico fueron ser diabético $(\mathrm{OR}=3,6)$, tener insuficiencia renal $(O R=5,4)$, en empleo de aminas vasoactivas $(O R=3,1)$. Se llega a la conclusión de que una medida de seguridad, para pacientes con estos factores, es la recolecta de muestras de sangre venosa y el empleo de hemogasometría para la verificación de la medida glucémica.

Palabras Clave: Insulina; glucemia; hipoglucemia; enfermería.

\section{INTRODUÇÃO}

Durante a cirurgia cardíaca os pacientes são submetidos a variações significativas na volemia, temperatura corporal, composição plasmática e fluxo sanguíneo tecidual com consequências fisiopatológicas importantes. Agressões como a circulação extracorpórea (CEC) e parada circulatória total contribuem para agravar ainda mais o desarranjo orgânico no intraoperatório'.

Esses pacientes estão sob estado de estresse fisiológico em virtude da gravidade de seu quadro clínico, que os predispõe a diversos distúrbios endócrinos, tais

'Doutoranda do Programa de Pós-Graduação em Enfermagem e Professora Convidada do Curso de Pós-Graduação em Enfermagem Intensivista da Universidade do Estado do Rio de Janeiro. Enfermeira do Instituto Nacional de Cardiologia. Rio de Janeiro, Brasil. E-mail: carinapaixao@gmail.com

"Doutoranda do Programa de Pós-Graduação em Enfermagem e Professora Convidada do Curso de Pós-Graduação em Enfermagem Intensivista da Universidade do Estado do Rio de Janeiro. Enfermeira do Instituto Nacional de Cardiologia. Rio de Janeiro, Brasil. E-mail: raquel.nepomuceno@gmail.com

IIIMestre pelo Programa de Pós-Graduação em Enfermagem e Professor Convidado do Curso de Pós-Graduação em Enfermagem Intensivista da Universidade do Estado do Rio de Janeiro. Enfermeiro do Instituto Nacional do Cancer. Rio de Janeiro, Brasil. E-mail: mana moura@yahoo.com

IV Professora Permanente do Programa de Pós-Graduação em Enfermagem e Coordenadora do Curso de Enfermagem Intensivista da Faculdade de Enfermagem da Universidade do Estado do Rio de Janeiro. Bolsista Pró-cientista. Rio de Janeiro, Brasil. E-mail: lolita.dopico@gmail.com 
como a hiperglicemia ${ }^{1}$. A hiperglicemia provoca efeitos deletérios micro e macrovasculares, como nefropatias, alterações do sistema imunológico, neuropatias, distúrbios hidroeletrolíticos e disfunções endotelias que predispõe à trombogênese. Considera-se difícil definir a incidência de hiperglicemia aguda, podendo ela variar entre 40 a $90 \%$, dependendo do limite utilizado para definir níveis anormais de glicemia ${ }^{1}$.

Dessa forma, nos últimos anos, o controle glicêmico intensivo obtido pela infusão contínua de insulina (ICI) passou a ocupar lugar de destaque no manejo dos pacientes críticos. A premissa é de que a manutenção da normoglicemia está associada a menores taxas de infecções e de falências orgânicas. A insulina é o único hormônio que faz esse transporte e sem ela as células ficam privadas de energia ${ }^{2}$.

De acordo com o Institute for Healthcare Improvement (IHI), a insulina pertence a uma das dez categorias de medicamentos considerados como Medicamentos de Alto Risco ou Medicamentos Potencialmente Perigosos (MPPs), o que significa que apresenta maior potencial para provocar lesão grave nos pacientes quando ocorre falha em seu processo de utilização. A insulina via intravenosa requer cuidados para garantir a eficiência e segurança de sua administração, pois possui efeitos colaterais, dentre os mais importantes, destaca-se a hipoglicemia que, se não for tratada, pode causar danos cerebrais irreversíveis ${ }^{3}$.

A hipoglicemia é considerada o principal evento adverso associado à insulinoterapia venosa contínua. A hipoglicemia grave ocorre em torno de $4 \%$ a $7 \%$ dos pacientes, e apesar de o risco de hipoglicemia não parecer muito elevado, o impacto que esse evento tem sobre o prognóstico torna obrigatório procurar soluções que possam contribuir para reduzir a ocorrência deste evento.

Na prática diária ainda se convive com freqüência com episódios de hipoglicemia durante a infusão venosa contínua de insulina, apesar do monitoramento pela enfermagem, que consiste em aferir por dígitopunção a glicemia de hora em hora, acompanhar os sinais vitais do paciente, além da manutenção de uma fonte contínua de glicose, tanto por nutrição enteral como parenterais, todas essas condutas guiadas guiadas por protocolos das unidades 4 .

No Brasil, cabe à enfermagem o preparo e a administração da solução de insulina na maioria dos hospitais, devendo, portanto, estar ela ciente dos aspectos que envolvem não somente o manejo da insulina, mas também seu monitoramento para evitar a ocorrência de hipoglicemia grave $(<50 \mathrm{mg} / \mathrm{dl})^{2}$.

Além destes aspectos, considera-se necesário o reconhecimento dos fatores predisponentes para hipoglicemia apontados na literatura, pois há situações em que, mesmo seguindo o protocolo com disciplina e aferindo-se a glicemia horária, uma parcela dos pacientes apresenta hipoglicemia, eventos imputados aos fatores predisponentes à hipoglicemia. Existem diversos fatores apontados na literatura, e por serem os mais citados, serão naqueles que esta pesquisa se fundamentará; são eles: a idade maior de 60 anos, a hipertensão arterial, diabetes mellitus, insuficiência renal, ausência de aporte calórico e o uso de aminas vasoativas ${ }^{4}$.

Este estudo objetivou descrever os fatores predisponentes para hipoglicemia, encontrados nos pacientes críticos no pós-operatório de cirurgia cardíaca que receberam ICI.

A contribuição desta publicação se fundamenta no fato de que cabe ao enfermeiro a supervisão e/ou administração de medicamentos, sendo sua responsabilidade o acompanhamento do paciente que recebe ICI, e, portanto conhecer quais os fatores predisponentes apresentados pelo paciente poderá contribuir para um planejamento de cuidados específico ao paciente, prevenindo eventos adversos que possam comprometer os resultados da terapia medicamentosa com insulina intravenosa, de forma a garantir um manejo seguro da insulina.

\section{REVISÃo DE LITERATURA}

Durante a cirurgia cardíaca os pacientes são submetidos a variações significativas na volemia, temperatura corporal, composição plasmática e fluxo sanguíneo tecidual com consequências fisiopatológicas importantes ${ }^{1}$. Agressões como a CEC e parada circulatória total, contribuem para agravar ainda mais o desarranjo orgânico no intraoperatório ${ }^{1}$.

As modificações endócrinas e as respostas imunológicas deflagradas levam a um conjunto de alterações metabólicas para proteger as principais funções fisiológicas ${ }^{2}$. As variações hormonais ao estresse causam liberação de excesso de hormônios como glucagon, catecolaminas e glicocorticóide, resultando em insuficiência de insulina para suprimir a gliconeogênese hepática, e comprometem a regulação insulina-captação de glicose. A hiperglicemia gerada, por muito tempo menosprezada e tida como um evento secundário, é hoje reconhecida como fator preditivo de mal prognóstico no paciente crítico ${ }^{5,6}$.

Dessa forma, nos últimos anos, o controle glicêmico intensivo obtido pela infusão contínua de insulina passou a ocupar lugar de destaque no manejo dos pacientes críticos. A premissa é de que a manutenção da normoglicemia está associada a menores taxas de infecções e de falências orgânicas. Sabe-se que a manutenção dos valores de glicemia entre 80 a $120 \mathrm{mg} / \mathrm{dl}$, diminui a morbidade, promove a redução da mortalidade em torno de $40 \%$ e proporciona também a redução no tempo de internação, além de diminuir a incidência de complicações e o desenvolvimento de disfunção orgânica?

Apesar de a insulina estar entre os MPPs, a terapia insulínica no controle glicêmico intensivo é uma possibilidade terapêutica de impacto na morbi-mortalidade 
dos pacientes críticos e sua implementação é de baixo custo, requerendo principalmente a devida capacitação e disciplina das equipes, principalmente da enfermagem ${ }^{8}$.

\section{Metodologia}

Realizada uma pesquisa com estrutura epidemiológica, coleta de dados documental retrospectiva de prontuários de pacientes que receberam infusão contínua de insulina de uma instituição hospitalar, sediada no Estado do Rio de Janeiro. Os dados foram coletados no período de março a outubro de 2014 e foram selecionados todos os prontuários de pacientes do pós-operatório e que utilizaram insulina intravenosa em 2012 e 2013 e atenderam aos critérios de inclusão, a saber: serem pacientes maiores de 18 anos; com no mínimo dois ou mais dias de internação; com registro preenchido da alta ou óbito e terem recebido ICI.

Foi considerada hipoglicemia grave quando a glicemia apresentava-se menor que $50 \mathrm{mg} / \mathrm{dL}$. Como fatores predisponentes foram especificados: diabetes, insuficiência renal; necessidade de aporte calórico e o uso de aminas vasoativas.

Como o hospital não tem o prontuário eletrônico, os dados foram coletados das evoluções clínicas, de balanço hídrico, exames laboratoriais e de prescrição médica.

Foram aplicadas as medidas estatísticas, descritivas, e considerou-se significativo um $\mathrm{p} \leq 0,05$. $\mathrm{O}$ valor $\mathrm{p}$ foi calculado pelo teste exato de Fisher, a fim de evidenciar diferenças estatísticas entre as variáveis para cada característica clínica. $\bigcirc$ valor $\mathrm{p}$ expressa a significância estatística relacionada à característica clínica e à ocorrência de hipoglicemia. Para quantificar o quão mais provável é a ocorrência da hipoglicemia nos pacientes com características clínicas para hipoglicemia, foi calculada a razão de risco (RR). Todos os preceitos éticos relativos ao desenvolvimento de pesquisas em seres humanos regulamentados pela Resolução brasileira 466/12 foram obedecidos no desenvolvimento deste estudo. $\mathrm{O}$ projeto, encaminhado ao Comitê de Pesquisa e Ética do Hospital Universitário Pedro Ernesto, foi aprovado sob o n $\mathrm{n}^{\circ} 3.083 / 2011$.

\section{Resultados}

Rastreados 550 prontuários do pós-operatório de cirurgia cardíaca, dos quais 168 pacientes receberam insulina intravenosa (ICI), em média durante três dias e desses 74 (44,04\%) pacientes apresentaram pelo menos um episodio de hipoglicemia grave hipoglicemia.

Entre os pacientes que usaram ICI, predominou o sexo masculino (60,11\%), idosos ( $\mathrm{md}=61,78$ anos), com sobrepeso, verificando-se uma mediana de 26,75 $\mathrm{Kg} / \mathrm{m} 2$ de Indice de Massa Corpórea (IMC). A maioria era hipertensa $(78,57 \%)$ e $52,38 \%$ eram isquêmicos. A maioria $(80,35 \%)$ dos pacientes teve a alta como destino da internação.

Entre os pacientes que apresentaram hipoglicemia, houve predomínio do sexo masculino $(29,16 \%)$, a maioria teve alta $(28,57 \%)$ e as patologias isquêmicas $(22,02 \%)$ foram a maior causa da cirurgia, Destaca-se que a única característica com diferença estatística significativa entre os grupos com e sem hipoglicemia foi ter tido IAM $(\mathrm{p}=0,01345)$, conforme a Tabela 1.

TABELA 1: Características clínicas dos pacientes com e sem hipoglicemia. RJ, 2012-201

\begin{tabular}{|c|c|c|c|c|c|c|c|}
\hline \multirow[t]{2}{*}{ Características } & \multicolumn{2}{|c|}{$\begin{array}{c}\text { Com Hipoglicemia } \\
(f=74)\end{array}$} & \multicolumn{2}{|c|}{$\begin{array}{c}\text { Sem Hipoglicemia } \\
(\mathrm{f}=94)\end{array}$} & \multicolumn{2}{|c|}{ Total $(f=168)$} & \multirow[t]{2}{*}{$p$ valor ${ }^{(*)}$} \\
\hline & $f$ & $\%$ & $f$ & $\%$ & $f$ & $\%$ & \\
\hline \multicolumn{8}{|l|}{ Sexo } \\
\hline Masculino & 49 & 29,16 & 52 & 30,95 & 101 & 60,11 & 0,2025 \\
\hline Feminino & 25 & 14,88 & 42 & 25 & 67 & 39,88 & \\
\hline \multicolumn{8}{|l|}{ HAS } \\
\hline Sim & 61 & 36,3 & 71 & 42,26 & 132 & 78,57 & 0,3727 \\
\hline Não & 13 & 7,73 & 23 & 13,69 & 36 & 21,42 & \\
\hline \multicolumn{8}{|l|}{ IAM } \\
\hline Sim & 5 & 2,97 & 20 & 11,9 & 25 & 14,88 & 0,01345 \\
\hline Não & 69 & 41,07 & 74 & 44,04 & 143 & 85,11 & \\
\hline \multicolumn{8}{|l|}{ Tabagismo } \\
\hline Sim & 18 & 10,71 & 23 & 13,69 & 41 & 24,4 & \\
\hline Não & 56 & 33,33 & 71 & 42,26 & 127 & 75,59 & $>0,99999$ \\
\hline \multicolumn{8}{|l|}{ Diagnóstico } \\
\hline Isquêmicos & 37 & 22,02 & 51 & 30,35 & 88 & 52,38 & 0,6945 \\
\hline Valvares & 23 & 13,69 & 35 & 20,83 & 58 & 34,52 & 0,5042 \\
\hline Características & $M(\sigma)$ & Md & $M(\sigma)$ & Md & \multicolumn{2}{|c|}{ Total } & \\
\hline Idade & $58,93(13,26)$ & 62 & $65,58(10,52)$ & 63,5 & 62,25 & 62,75 & 0,7983 \\
\hline Peso & $70,1(10,06)$ & 68 & $70,05(11,49)$ & 73 & 70,07 & 70,5 & 0,2604 \\
\hline Altura & $160,3(7,68)$ & 163 & $161(7,86)$ & 162 & 160,65 & 162,5 & $>9999$ \\
\hline
\end{tabular}


Na Tabela 2, apresenta-se a distribuição dos fatores predisponentes citados pela literatura que podem favorecer a hipoglicemia nos pacientes que utilizaram infusão contínua de insulina.

Na população estudada, os fatores predisponentes para hipoglicemia com significado estatístico foram ser diabético, ter insuficiência renal, estar em uso de aminas vasoativas .

No Tabela 3, encontram-se o Odds Ratio (OR) e o respectivo intervalo de confiança, para cada fator predisponente encontrado, constatando-se que pacientes com diabetes têm 3,6 mais chances de apresentar hipoglicemia do que pacientes sem diabetes. A insuficiência renal está associada a uma chance 5,4 vezes maior de hipoglicemia, e o uso de vasoaminas aumenta em até três vezes a chance de hipoglicemia

\section{Discussão}

Na população pesquisada foram encontrados os seguintes fatores predisponentes para hipoglicemia: diabetes $(\mathrm{OR}=3,6)$, insuficiência renal $(\mathrm{OR}=5,4)$ e uso de vaosaminas $(\mathrm{OR}=3,1)$.

\section{Insuficiência renal}

Pacientes com insuficiência renal representaram quase $15,47 \%$ da população , dos quais $12 \%$ apresenta- ram hipoglicemia, havendo cinco vezes mais chance de pacientes com insuficiência renal virem a apresentar hipoglicemia.

Os rins são o principal sítio de degradação de insulina; de fato, as necessidades de insulina dos pacientes diabéticos caem conforme a insuficiência renal se instala. Assim, no paciente que usa insulina ou hipoglicemiante oral, a ocorrência de hipoglicemia pode ser explicada por este mecanismo e tratada com a correção da insulina. Contudo, alguns pacientes com insuficiência renal dialítica podem apresentar hipoglicemias espontâneas. Esses pacientes apresentam aumento na meia vida da insulina circulante, e não ocorre a neoglicogênese renal que colabora com $45 \%$ da produção de glicose. A hipoglicemia, em indivíduos com insuficiência renal que façam terapêutica dialítica e usem insulina, também é resultante da diminuição da depuração da insulina e da redução da mobilização dos precursores neoglucogênicos na insuficiência renal ${ }^{9-11}$.

A hipoglicemia relacionada à insuficiência renal envolve um ou mais entre vários mecanismos, como a gliconeogênese hepática deficiente, glicogenólise alterada, gliconeogênese renal diminuída, degradação e clereance renal da insulina prejudicadas, desnutrição, e, em certos casos, deficiência de hormônios contrarreguladores, como deficiência de catecolaminas e

TABELA 2 : Fatores predisponentes para hipoglicemia dos grupos estudados. Rio de Janeiro - 2014

\begin{tabular}{|c|c|c|c|c|c|c|c|}
\hline \multirow[t]{2}{*}{$\begin{array}{c}\text { Fatores } \\
\text { Predisponentes }\end{array}$} & \multicolumn{2}{|c|}{$\begin{array}{c}\text { Com Hipoglicemia } \\
(f=74)\end{array}$} & \multicolumn{2}{|c|}{$\begin{array}{c}\text { Sem Hipoglicemia } \\
(\mathrm{f}=94)\end{array}$} & \multicolumn{2}{|c|}{ Total $(f=168)$} & \multirow[t]{2}{*}{ p valor $\left.{ }^{*}\right)$} \\
\hline & $f$ & $\%$ & $f$ & $\%$ & $f$ & $\%$ & \\
\hline \multicolumn{8}{|l|}{ Diabetes } \\
\hline Sim & 42 & 25 & 25 & 14,88 & 67 & 39,88 & 0,0001342 \\
\hline Não & 32 & 19,04 & 69 & 41,07 & 101 & 60,11 & \\
\hline \multicolumn{8}{|l|}{ Insuficiência Renal } \\
\hline Sim & 20 & 11,9 & 6 & 3,57 & 26 & 15,47 & 0,0004949 \\
\hline Não & 54 & 32,14 & 88 & 52,38 & 142 & 84,52 & \\
\hline \multicolumn{8}{|l|}{ Aporte Calórico } \\
\hline Sim & 70 & 41,66 & 89 & 52,97 & 159 & 94,64 & \\
\hline Não & 4 & 2,38 & 5 & 2,97 & 9 & 5,35 & 0,62026 \\
\hline \multicolumn{8}{|l|}{ Aminas } \\
\hline Sim & 48 & 28,57 & 35 & 20,83 & 83 & 49,4 & 0,000628 \\
\hline Não & 26 & 15,47 & 59 & 35,11 & 85 & 50,59 & \\
\hline
\end{tabular}

(*) O p valor foi calculado pelo teste exato de Fisher considerando significativo se $p<0,05$.

Fonte: Prontuários de pacientes, hospital X.

TABELA 3: Fatores predisponentes para hipoglicemia. Rio de janeiro, 2014. ( $N=74)$

\begin{tabular}{lcc}
\hline Fatores predisponentes & p valor $^{(*)}$ & OR e Intervalo de confiança \\
\hline Diabetes & 0.0001342 & $3.623(1.894-6.93)$ \\
Insuficiencia Renal & 0.0004949 & $5.432(2.053-14.37)$ \\
Uso de Aminas & 0.0006280 & $3.112(1.65-5.87)$ \\
\hline
\end{tabular}

(*) O p valor foi calculado pelo teste exato de Fisher considerando significativo se $p<0,05$. 
glucagon $^{12,13}$. Quando esses pacientes fazem diálise, eles apresentam uma maior sensibilidade à insulina através do aumento da liberação de moléculas inflamatórias, como interleucinas, levando a um maior risco a hipoglicemia.. Neste caso, faz-se uma correção e adaptação da dose de insulina ${ }^{14}$.

\section{Diabetes}

A presença de Diabetes Mellitus foi uma característica apresentada por quase $40 \%$ da população dos quais 25\% $(n=42)$ apresentaram hipoglicemia , havendo três vezes mais chance de pacientes com diabetes virem a apresentar hipoglicemia.

A hipoglicemia pode ser provocada por vários fatores dentro das UTIs, como, erro na dose de insulina intravenosa, mudanças de medicação, alteração nos locais de verificação da glicemia, ausência de aporte calórico e diminuição da necessidade de insulina, após a saída de situação de estresse cirúrgico ${ }^{15}$.

O controle deficiente da glicemia está relacionado a piores desfechos hospitalares, não apenas no paciente diabético, mas também no não-diabético. $\mathrm{O}$ estresse induzido pelo procedimento cirúrgico e pela circulação extracorpórea na cirurgia cardíaca pode levar a importante hiperglicemia. Níveis de glicemia maiores que 150 $\mathrm{mg} / \mathrm{dl}$ no período operatório e superiores a $350 \mathrm{mg} / \mathrm{dl}$ durante a circulação extracorpórea foram relacionados a aumento das complicações cardiovasculares, respiratórias, infecciosas, neurológicas e renais. Pacientes com deficiente controle glicêmico intraoperatório podem apresentar sete vezes mais chances de complicações em relação aos demais. As hipoglicemias, principalmente em diabéticos, induzem deficiente mecanismo de contrarregulação, com diminuição de liberação de adrenalina e dos sintomas de alarme. Em função da má nutrição, principalmente em UTIs a reserva de glicogênio hepático poderá ficar comprometida, o que, em associação ao déficit de catecolaminas e cortisol, poderá também predispor o paciente a uma hipoglicemia ${ }^{16}$.

\section{Vasoaminas}

Quase a metade dos pacientes $(49,4 \%)$ fez uso de vasoaminas, dos quais 28,57\% apresentaram hipoglicemia, havendo três vezes mais chances de esses pacientes virem a apresentar hipoglicemia.

Devido à instabilidade hemodinâmica que pode acontecer no pós-operatório imediato de cirurgia cardíaca, é frequente o paciente precisar de drogas vasoativas, assim como, de ventilação mecânica, suporte circulatório, com consequente aumento do tempo de permanência na terapia intensiva ${ }^{17}$.

Pacientes hipoperfundidos nos estados de choque, com edema periférico e em uso de medicamentos vasoconstrictores podem apresentar resultados glicêmicos erroneamente baixos pelo comprometimento da qualidade da amostra capilar.
Diante destas características, recomenda-se a coleta de amostra venosa. A amostra venosa é a mais confiável, seguindo-se do sangue arterial e por último a capilar. Em virtude da influência de vários fatores, a medida glicêmica pelo sangue capilar através de glicosímetro não é recomendada como único método em pacientes hemodinamicamente instáveis e que utilizam vasoaminas ${ }^{18}$.

Recomenda-se que a enfermagem solicite a confirmação laboratorial dos valores extremos de hipo ou hiperglicemia. Contudo, sabe-se das dificuldades dessa medida, como a disponibilidade do laboratório central em responder com prontidão a esta demanda e a definição de qual valor glicêmico indicará a necessidade dessa confirmação. Assim, acredita-se que repetir a mensuração glicêmica através do glicosímetro com uma amostra de origem diferente, diante de valores de glicemia menores que $60 \mathrm{mg} / \mathrm{dl}$ ou maiores que 250 $\mathrm{mg} / \mathrm{dl}$, usando hemogasômetros seja uma medida mais viável nas unidades intensivistas brasileiras ${ }^{19}$.

No contexto da terapia intensiva, é necessário que enfermeiros contribuam para a diminuição dos episódios de hipoglicemia grave, beneficiando pacientes e instituições hospitalares nas questões de segurança do paciente que recebe insulina intravenosa ${ }^{20}$.

\section{Conclusão}

Ao associar a presença de fatores predisponentes à ocorrência de hipoglicemia foram investigados pacientes com mais de 60 anos, com diabetes, com insuficiência renal, com aporte calórico e em uso de aminas vasoativas.

Os fatores predisponentes que revelavam associação positiva com a presença de hipoglicemia foram a insuficiência renal, o diabetes e o uso de vasoaminas, indicando que a presença do fator predisponente aumentou a chance de hipoglicemia.

Portanto, para garantir o uso seguro na infusão de insulina intravenosa, recomenda-se que as ações de enfermagem incorporem a identificação dos fatores predisponentes de cada paciente. $\mathrm{O}$ enfermeiro deve planejar os procedimentos de forma a evitar a demora ou atraso na execução de exames que requeiram jejum, realizar a oferta alimentar e a medição da glicemia capilar em horários determinados.

Nesse sentido, entende-se que o enfermeiro, ao cuidar de um paciente que recebe infusão contínua de insulina, deve conhecer essas principais características para a ocorrência de hipoglicemia. Devido à natureza multifatorial da ocorrência de hipoglicemia, o conhecimento dos fatores predisponentes é fundamental para a adoção de medidas preventivas e terapêuticas.

As limitações do estudo referem-se principalmente à perda de dados em virtude da qualidade dos prontuários, sendo um fator insuperável, não somente 
no que tange aos registros incompletos, mas também às informações nem sempre confiáveis, obrigando ao cruzamento de vários dados do paciente para o aproveitamento do caso clínico.

Recomenda-se a realização de novos estudos para testar quais cuidados de enfermagem seriam efetivos como barreira a episódios de hipoglicemia; quais fatores sinalizam o risco da mensuração glicêmica não detectar a hipoglicemia e quais são os fatores predisponentes que, quando combinados, indicam o paciente com maior ou menor suscetibilidade para a ocorrência de evento adverso durante a ICI.

\section{REFERÊNCIAS}

1.Magalhães FGC. A insulinoterapia intensiva nos diferentes contextos de hiperglicemia em âmbito hospitalar. Portugal: Instituto de Ciências Biomédicas Abel Salazar Largo; 2010.

2.Goodman \& Gilman. Manual de farmacologia e terapêutica. 20a ed. Porto Alegre (RS): AMGH; 2010.

3.Intitute for Safe Medication Pratices. ISMP's listo $f$ high-alert medications. [Internet]. ISMP, 2012. [cited in 2014 Mar 20] Disposable in: http://www.ismp.org/tools/ highalertmedications.pdf

4.Stamou SC, Nussbaum M, Carew JD, Skipper E, Robicsek F, Lobdellet KW. Hypoglycemia with intensive insulin therapy after cardiac surgery: predisposing factors and association with mortality. Thorac Cardiovasc Surg [periódico na internet]. 2012 [cited in 2014 Mar 20]; 32:82-97 . Disponível em: http://www.ncbi.nlm.nih.gov/ pubmed/?term $=$ Stamou $\% 2 \mathrm{C}+$ Sotiris $+\mathrm{C} . \% 3 \mathrm{~B}+$ Nussba um $\% 2 \mathrm{C}+$ Macy\%3BCarew\%2C+John +D.\%3B + Dunn $\% 2 \mathrm{C}+$ Kelli\%3BSkipper\%2C+Eric\%3B+Robicsek\%2C + Francis\%3B + Lobdell\%2C+Kevin +W.

5.Guyton AC, Hall JE. Tratado de fisiologia médica. 10 ed. Rio de Janeiro: Guanabara Koogan; 2009.

6.Sociedade Brasileira de Diabetes. Controle da hiperglicemia intra-hospitalar em pacientes críticos e não críticos. Posicionamento Oficial SBD n ${ }^{\circ} 02 / 2011$. [citado em 20 dez 2014]. Disponível em: http://www.nutritotal. com.br/diretrizes/files/228posicionamento_sbd_hiperglicemia.pdf.

7.Diretrizes da SBC sobre angina instável e infarto agudo do miocárdio sem supra desnivelamento do segmento ST (II edição, 2007) - Atualização 2013. Arq Bras Cardiol. 2014; 102 (3Supl.1).

8.Via MA, Scurlock C, Adams DH, Weiss AJ, Mechanick JI. Impaired postoperative hyperglycemic stress response associated with increased mortality in patients in the cardiothoracic surgery intensive care unit. Endocr
Pract [Internet]. 2010 [cited in 2014 Feb 05]; 16:798804. Disposable in: http:/www.ncbi.nlm.nih.gov/pubmed/20350912

9. Wiener RS, Wiener DC, Larson RJ. Benefits and Risks of Tight Glucose Control in Critically Ill Adults A Metaanalysis. JAMA [Internet]. 2008 [cited in 2014 Feb 05]; 300: 933-44. Disponível em: http://www.ncbi.nlm.nih. gov/pubmed/18728267

10.Egi M, Bellomo R, Stachowski E, French CJ, Hart GK, Taori G, et al. The interaction of chronic and acute glycemia with mortality in critically ill patients with diabetes. [Internet]. 2007 [cited in 2014 Feb 05]; 35(9):S503-7. Disponível em: http://www.ncbi.nlm.nih. gov/pubmed/17713400

11.Carvalho D, Esteves C, Neves C. A Hipoglicemia no Diabético: controvérsia na avaliação, à procura das suas implicações. Acta Médica Portuguesa. 2012; 25: 454-60. 12.Morais GFC, Soares MJGO, Costa MML, Santos IBC. O diabético diante do tratamento, fatores de risco e complicações crônicas. Rev enferm UERJ. 2009; 17: 240-5. 13.Silva WO. Controle glicêmico em pacientes críticos em UTI. Revista HUPE. 2013; 12 (3): 47-56.

14.Soares GMT, Ferreira DCS, Gonçalves MPC, Alves TGS, David FL. Prevalência das principais complicações pós-operatórias em cirurgias cardíacas. Rev Bras Cardiol. 2011; 24(3):139-46.

15.Bastos MG, Bregman R, Kirsztajn GM. Doença renal crônica: frequente e grave, mas também prevenível e tratável. Rev Assoc Med Bras. 2010; 56: 248-53.

16.Sociedade Brasileira de Diabetes. Controle da hiperglicemia intra-hospitalar em pacientes críticos e não críticos. Posicionamento Oficial SBD n 02 /2011. [citado em 05 dez 2014]. Disponível em: http://www.nutritotal.com.br/ diretrizes/files/228posicionamento_sbd_hiperglicemia.pdf. 17.Preiser JC, Devos P. Clinical experience with tight glucose control by intensive insulin therapy. Crit Care Med [periódico na internet]. 2007 [cited in 2014 Dec 05]; 35:503-7. Disposable in: http://www.ncbi.nlm.nih. gov/pubmed/17713400

18.Ellahham S. Insulin therapy in critically ill patients. Vasc Health Risk Manag [periódico na internet]. 2010 [cited in 2014 Dec 05]; 6:1089-10.1 Disposable in: http:// www.ncbi.nlm.nih.gov/pubmed/21191429

19.Melissa Pitrowsky M, Shinotsuka CR, Soares M, Salluh JIF. Glucose control in critically ill patients in 2009: no alarms and no surprises. Rev Bras Ter Intensiva [periódico na internet]. 2009 [cited in 2014 Dec 05]; 21:310-4. Disposable in: http://www.scielo.br/scielo. php? script $=$ sci_arttext\&pid $=$ S0103-507X200900030 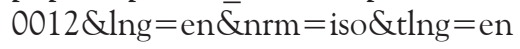

20.Silva LD. Segurança e qualidade nos hospitais brasileiros Rev enferm UERJ. 2013; 21:425-6. 